\title{
Chirality of structure and magnetism in the magnetoelectric compound $\mathrm{Cu}_{2} \mathrm{OSeO}_{3}$
}

\author{
V. Dyadkin,,${ }^{1,2,{ }^{*}}$ K. Prša,${ }^{3}$ S. V. Grigoriev, ${ }^{2,4}$ J. S. White,,${ }^{3,5}$ P. Huang, ${ }^{3}$ H. M. Rønnow, ${ }^{3}$ A. Magrez, ${ }^{6}$ \\ C. D. Dewhurst, ${ }^{7}$ and D. Chernyshov ${ }^{1}$ \\ ${ }^{1}$ Swiss-Norwegian Beamlines at the European Synchrotron Radiation Facility, 38000 Grenoble, France \\ ${ }^{2}$ Petersburg Nuclear Physics Institute, 188300 Gatchina, Saint Petersburg, Russia \\ ${ }^{3}$ Laboratory for Quantum Magnetism, Ecole Polytechnique Fédérale de Lausanne (EPFL), CH-1015 Lausanne, Switzerland \\ ${ }^{4}$ Saint Petersburg State University, Ulyanovskaya 1, 198504 Saint Petersburg, Russia \\ ${ }^{5}$ Laboratory for Neutron Scattering, Paul Scherrer Institut, CH-5232 Villigen, Switzerland \\ ${ }^{6}$ Institute of Condensed Matter Physics, Ecole Polytechnique Fédérale de Lausanne (EPFL), CH-1015 Lausanne, Switzerland \\ ${ }^{7}$ Institute Laue-Langevin, 38042 Grenoble Cedex 9, France
}

(Received 27 December 2013; published 21 April 2014)

\begin{abstract}
Small-angle diffraction of polarized neutrons and resonant contribution to diffraction of synchrotron radiation have been applied to prove chirality of the crystal and magnetic structures of the magnetoelectric insulator $\mathrm{Cu}_{2} \mathrm{OSeO}_{3}$. Similarly to other chiral magnets with $P 2_{1} 3$ crystal structure the corresponding chiralities are linked to each other via the phenomenological Dzyaloshinskii-Moriya interaction. The crystal and magnetic structures for $\mathrm{Cu}_{2} \mathrm{OSeO}_{3}$ have the same chirality as is observed for $\mathrm{MnSi}, \mathrm{Mn}_{1-x} \mathrm{Fe}_{x} \mathrm{Si}$, and $\mathrm{MnGe}$. However, the combination of chiralities is opposite to that proposed from a recent theoretical consideration. The chiral link between structure and magnetism previously found for metallic compounds is also proved to exist for an insulator $\left(\mathrm{Cu}_{2} \mathrm{OSeO}_{3}\right)$, allowing us to conclude that the conducting electrons play no role in a possible common microscopic mechanism of this specific magnetolattice interaction.
\end{abstract}

DOI: 10.1103/PhysRevB.89.140409

PACS number(s): 75.40.-s, 61.05.cp, 61.05.fg

The coupling between structural and magnetic properties results in many interesting and technologically important phenomena: the magnetoelastic effect, magnetostriction, and magnetoelectric coupling may serve as important examples [1]. Another type of coupling is possible if a chiral magnetic order forms on a chiral crystal lattice [2]. Such a chiral link has been determined experimentally for the well-known itinerant chiral magnets that display the cubic space group $P 2{ }_{1} 3, \mathrm{MnSi}$ [3-5], $\mathrm{Mn}_{1-x} \mathrm{Fe}_{x} \mathrm{Si}$ [6], $\mathrm{Fe}_{1-x} \mathrm{Co}_{x} \mathrm{Si}$ [7], $\mathrm{Mn}_{1-x} \mathrm{Fe}_{x} \mathrm{Ge}$ [8] for both the helically ordered ground state and also for the so-called skyrmion phase [9]. Detailed studies even show that the crystal chirality can be controlled during the crystal growth process [7]. Importantly, the magnetic chirality is intrinsically dependent on the structural one via the Dzyaloshinskii-Moriya interaction (DMI) [10] since it is the chemical composition which sets the sign of the DMI [8]. The microscopic mechanism underlying the chiral magnetolattice coupling, and which describes the chemical control of the magnetic chirality, is still, however, unclear. The possible explanation may be related to magnetotransport properties as discussed in Ref. [5]. Alternatively, the effect of next-nearest neighbors and outer shells on the sign of the DMI was suggest in the theoretical analysis of Ref. [11].

$\mathrm{Cu}_{2} \mathrm{OSeO}_{3}$ is another compound with the $P 2_{1} 3$ chiral cubic structure that has a magnetoelectric coupling together with the DMI [12-14]. It shows magnetic chirality and, furthermore, a magnetic field-temperature $(H-T)$ phase diagram that is very similar to $\mathrm{MnSi}$ and related compounds [15,16]. However, unlike the other compounds $\mathrm{Cu}_{2} \mathrm{OSeO}_{3}$ is an insulator. Nonetheless, it has the same magnetic phases as MnSi: a helical phase, a single domain conical phase, an induced ferromagnetic phase (ferrimagnetic in $\mathrm{Cu}_{2} \mathrm{OSeO}_{3}$ ), and also

*diadkin@esrf.fr the so-called A phase. The last phase exists in a small pocket of magnetic field and temperature and contains the skyrmion lattice, which is found for the first time in insulating materials. It was recently shown that in the two-dimensional limit (thin film) the skyrmion phase in $\mathrm{Cu}_{2} \mathrm{OSeO}_{3}$ becomes stable over a wider range of $H$ and $T$ [17] and the magnetoelectric coupling of this compound allows for the rotation of the skyrmion lattice as a function of applied electric field [18]. However, the correlation between the structural and magnetic chiralities has not yet been tested for $\mathrm{Cu}_{2} \mathrm{OSeO}_{3}$. Such a study is especially interesting bearing in mind that the insulating character of the material allows us to exclude any contribution due to conducting electrons.

The crystal structure of $\mathrm{Cu}_{2} \mathrm{OSeO}_{3}$ has been studied before $[19,20]$ but has never been revisited using modern crystallographic tools that allow the establishment of the absolute structure via the refinement of so-called Flack parameter [21-23]. Such analysis is based on the difference between Friedel pairs $[I(h k l)-I(\bar{h} \bar{k} \bar{l})]$ due to a resonant contribution in the x-ray scattering amplitudes. A measured difference provides direct information concerning the ratio between two opposite-handed structural domains for chiral space groups. A Flack parameter equal to zero corresponds to a single domain of the chiral structure (enantiopure) with the atomic coordinates calculated during such a refinement providing an absolute (i.e., with fixed handedness) structural configuration.

The ground state magnetic structure of $\mathrm{Cu}_{2} \mathrm{OSeO}_{3}$ is a proper spin helix [15]. The chiral sense of the magnetic modulation can be unambiguously identified using polarized neutron scattering [24]. This is done by determining the difference between two intensities collected at the same point of reciprocal space for two opposite neutron polarizations (along and opposite to a magnetic guide field) of the incident neutron beam; the method has been successfully implemented 
before for $\mathrm{MnSi}$ [3-5], $\mathrm{Mn}_{1-x} \mathrm{Fe}_{x} \mathrm{Si}$ [6], $\mathrm{Fe}_{1-x} \mathrm{Co}_{x} \mathrm{Si}$ [7], Ho [25], and Dy/Y multilayers [26]. Due to the large helical period for the title compound [15] $(d=646 \pm 45 \AA$ ) the first diffraction peak is expected at $q=|\mathbf{k}| \approx 0.01 \AA^{-1}$, where $\mathbf{k}$ is the helical wave vector which value is $k=2 \pi / d$. The $q$ range is most easily accessible using the small-angle neutron scattering (SANS) technique.

In this Rapid Communication, we present the results of synchrotron single crystal diffraction, and polarized SANS experiments done on the same single crystal. We show that in both experiments the diffracting structure appears as an enantiopure object. On the basis of a structural analysis, we define the sense of the absolute crystal structure and compare it with the sense of the magnetic helix with the latter being set according to a left-handed MnSi sample taken as a standard.

$\mathrm{Cu}_{2} \mathrm{OSeO}_{3}$ single crystals were synthesized via chemical vapor transport redox reactions. $\mathrm{CuO}$ and $\mathrm{SeO}_{2}$ were sealed in a silica ampule (of length $20 \mathrm{~cm}$ and diameter $3.6 \mathrm{~cm}$ ) together with 200 mbar of $\mathrm{HCl}$ (electronic grade purity). The ampule was subsequently placed in a two-zone furnace with chargeand growth-zone temperatures of 880 and $800 \mathrm{~K}$, respectively. After three weeks, the transport process was completed and dark green crystals of $\mathrm{Cu}_{2} \mathrm{OSeO}_{3}$ were collected.

The chirality of the magnetic helix in our $\mathrm{Cu}_{2} \mathrm{OSeO}_{3}$ single crystal was measured using the D22 SANS machine (ILL, Grenoble, France); the initial polarization of the neutron beam was $P_{0}=0.84$. The wave length of the neutron beam was set to $8.15 \AA$ and the $\mathrm{Cu}_{2} \mathrm{OSeO}_{3}$ single crystal was aligned such that the beam went parallel to the [001] crystallographic direction and the magnetic Bragg peaks $(\delta, 0,0), \delta=0.01 \AA^{-1}$, were in a range accessible with the detector. The magnetic field was applied normal to the beam (for further details see Ref. [27]) and the temperature was controlled using an ILL Orange cryostat.

The handedness of a magnetic helix can be unambiguously characterized by comparing its diffraction pattern to that for a standard reference sample. We have used an $\mathrm{MnSi}$ single crystal as such a reference standard since it has been thoroughly characterized before [5] and its chirality has also been confirmed by different techniques $[9,28]$. MnSi single crystals can be grown in both left- and right-handed forms, that respectively result in left- and right-handed magnetic helices at low temperature [7]; a left-handed single crystal was used in our experiment.

Figure 1 shows typical SANS diffraction patterns from both $\mathrm{MnSi}$ and $\mathrm{Cu}_{2} \mathrm{OSeO}_{3}$. As one can see, the reference sample shows a maximum of the scattering intensity at the right side of the detector when the initial polarization of the neutron beam $P_{0}$ is along the magnetic guide field [Fig. 1(a)] and at the left side for the opposite neutron polarization [Fig. 1(b)]. Clearly, the $\mathrm{Cu}_{2} \mathrm{OSeO}_{3}$ sample displays the opposite behavior. Since the $\mathrm{MnSi}$ sample is known to be $100 \%$ magnetically left-handed [3-5], we define the magnetic chirality of the $\mathrm{Cu}_{2} \mathrm{OSeO}_{3}$ sample as right-handed.

Using the SANS data we can estimate the chiral magnetic fraction in $\mathrm{Cu}_{2} \mathrm{OSeO}_{3}, \gamma$, as

$$
\gamma=\frac{P_{s}}{P_{0}} \cos \psi
$$

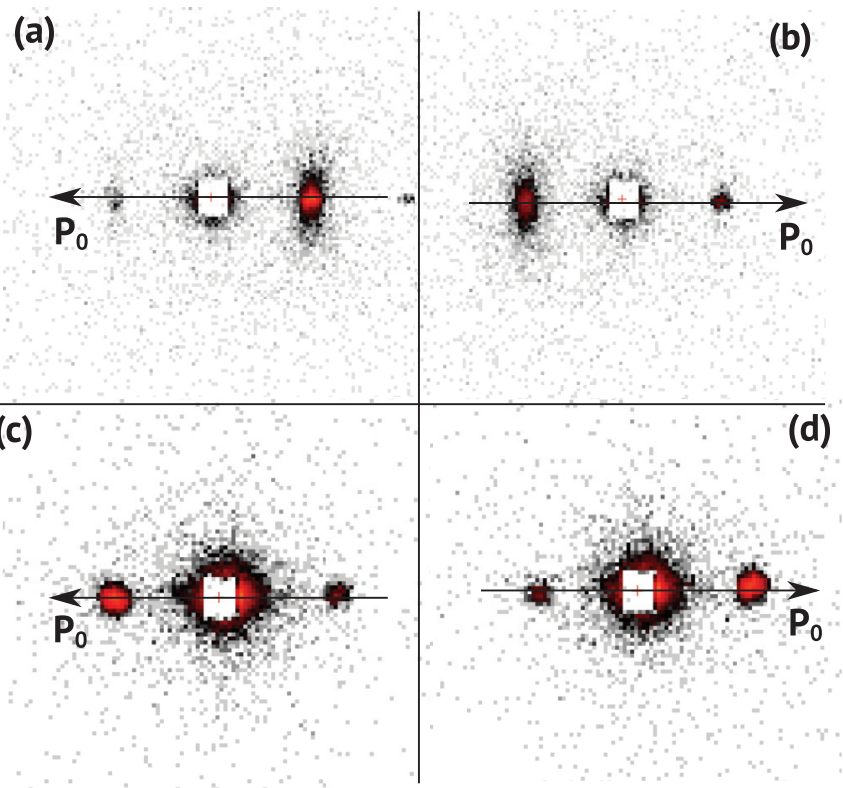

FIG. 1. (Color online) Maps of the polarized SANS intensities of $\mathrm{MnSi}[(\mathrm{a}),(\mathrm{b})]$ at $T=15 \mathrm{~K}$ and $\mathrm{Cu}_{2} \mathrm{OSeO}_{3}$ [(c), (d)] at $T=35 \mathrm{~K}$ for the polarization $P_{0}$ along the guide field [(a), (c)] and opposite to it [(b), (d)]. The applied magnetic field is $B=80 \mathrm{mT}$ for $\mathrm{MnSi}$ and $50 \mathrm{mT}$ for $\mathrm{Cu}_{2} \mathrm{OSeO}_{3}$.

where $\psi$ is the azimuthal angle between the scattering vector $\mathbf{q}$ and $\mathbf{P}_{0}$. The sample polarization $P_{s}$ is calculated as

$$
P_{s}=\frac{I\left(+\mathbf{P}_{0}, \mathbf{Q}\right)-I\left(-\mathbf{P}_{0}, \mathbf{Q}\right)}{I\left(+\mathbf{P}_{0}, \mathbf{Q}\right)+I\left(-\mathbf{P}_{0}, \mathbf{Q}\right)},
$$

where $I\left(+\mathbf{P}_{0}, \mathbf{Q}\right)$ and $I\left(-\mathbf{P}_{0}, \mathbf{Q}\right)$ are the integrated scattering intensities measured at the same point of reciprocal space $\mathbf{Q}=\mathbf{k}$ but for opposite initial polarizations.

The typical intensity dependence as a function of the magnetic field at the point $\mathbf{Q}=\mathbf{k}$ is shown in Fig. 2. Here, $B_{C 1}$ is the first critical magnetic field, above which the cubic anisotropy is overcome and the magnetic helices change their orientation from $q \|\langle 100\rangle$ to the direction parallel to $\mathbf{B}$. $B_{C 2}$

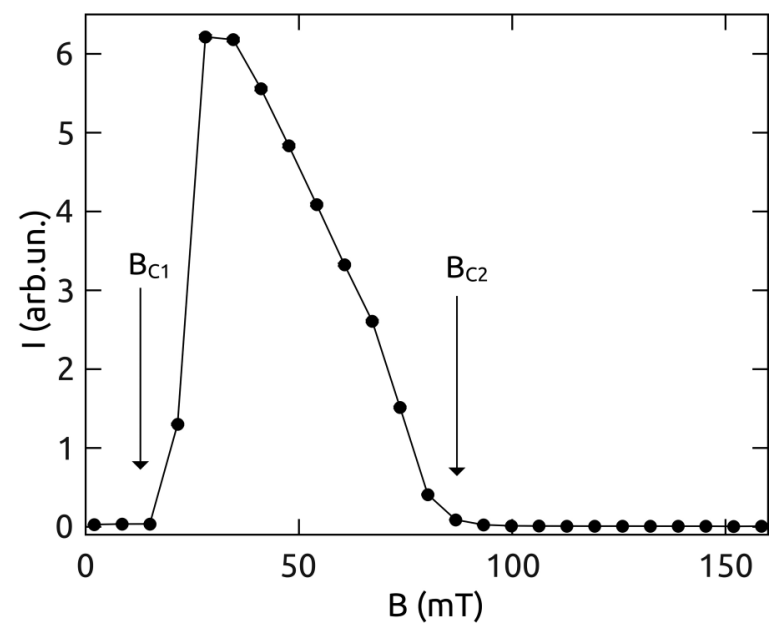

FIG. 2. Intensity of $\mathrm{Cu}_{2} \mathrm{OSeO}_{3}$ at $\mathbf{q} \| \mathbf{P}_{0}$ as a function of the magnetic field $B$ at $T=35 \mathrm{~K}$. The error bars are smaller than points. 


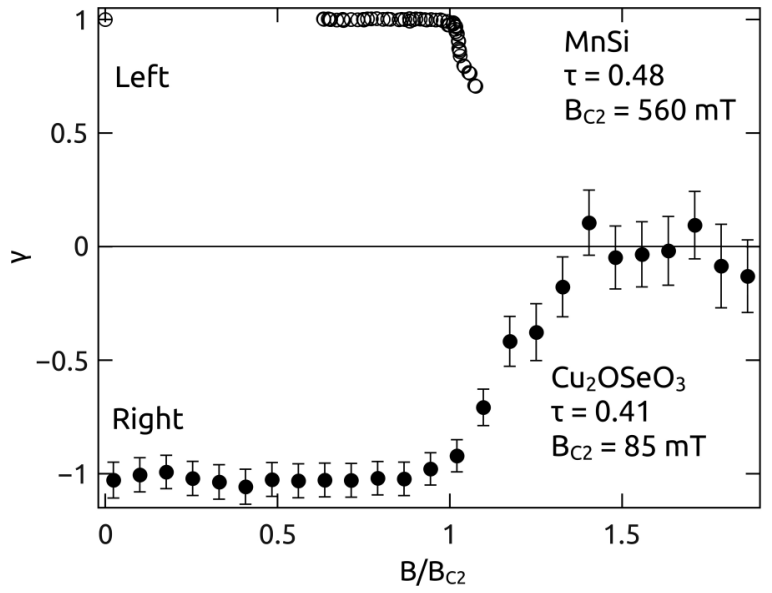

FIG. 3. The magnetic chiral fraction $\gamma$ as a function of the relative magnetic field $B / B_{C 2}$ for $\mathrm{MnSi}$ (top) and $\mathrm{Cu}_{2} \mathrm{OSeO}_{3}$ (bottom). The 1 and -1 values correspond to the enantiopure state with left and right handedness, respectively. $\tau=\left(T_{c}-T\right) / T_{c}$, MnSi data are adopted from Ref. [29].

is the second critical magnetic field, above which the sample becomes ferrimagnetic.

Figure 3 shows the dependency of the magnetic chiral fraction on the relative magnetic field $B / B_{C 2}$ for the standard $\mathrm{MnSi}$ sample (top panel) and the $\mathrm{Cu}_{2} \mathrm{OSeO}_{3}$ sample (bottom panel). $\mathrm{Cu}_{2} \mathrm{OSeO}_{3}$ is discovered to be enantiopure within uncertainty (the ratio of volume fractions of domains with opposite to MnSi chirality is not more than $\approx 1 / 100$ ).

The synchrotron single crystal diffraction data [30] agree well with previously reported $[13,19,20]$ structure. The full sphere of Bragg reflections was used to refine the Flack parameter $\left[\eta_{f}=-0.028(12)\right]$. According to Ref. [31] such a result corresponds to a chiral crystal that is not twinned by inversion. Therefore, the absolute structure (Tables I and II) can be used to set the sense of chirality for a given $\mathrm{Cu}_{2} \mathrm{OSeO}_{3}$ crystal, and it is done the following way. First, we note that both the $\mathrm{Cu}_{2} \mathrm{OSeO}_{3}$ and $\mathrm{MnSi}$ crystal structures display the same $P 2{ }_{1} 3$ space group and also a magnetic ion occupying the same Wyckoff position; both $\mathrm{Cu}(1)$ (Table II) and $\mathrm{Mn}$ sit at the 4 a position $(x, x, x / 0.5+x, 0.5-x,-x /-x, 0.5+$ $x, 0.5-x / 0.5-x,-x, 0.5+x)$. Second, as has been shown for $\mathrm{MnSi}$, the chirality of the crystal structure can be characterized by the value of $x$ [2]. Thus $x \approx 0.137$ corresponds to the

TABLE I. Crystal data and structure refinement for $\mathrm{Cu}_{2} \mathrm{OSeO}_{3}$.

\begin{tabular}{lc}
\hline \hline Temperature & $293 \mathrm{~K}$ \\
Crystal system & cubic \\
Space group & $P 2_{1} 3$ \\
Wavelength & $0.69156 \AA$ \\
Unit cell dimension $a$ & $8.9080(5) \AA$ \\
$\theta$ range for data collection & $\theta_{\min }=3.147^{\circ}, \theta_{\max }=32.252^{\circ}$ \\
& $-13 \leqslant h \leqslant 13$ \\
Limiting indices & $-11 \leqslant k \leqslant 11$ \\
& $-13 \leqslant l \leqslant 13$ \\
Reflections collected $/$ unique & $5255 / 886\left[R_{\text {int }}=0.0367\right]$ \\
Final $R$ indices $[I>2 \sigma(I)]$ & $R_{1}=0.0312, w R_{2}=0.0685$ \\
Absolute structure parameter & $-0.028(12)$ \\
\hline \hline
\end{tabular}

TABLE II. Atomic coordinates and Wyckoff positions (WP) for $\mathrm{Cu}_{2} \mathrm{OSeO}_{3}$.

\begin{tabular}{lcccc}
\hline \hline & WP & $x$ & $y$ & $z$ \\
\hline $\mathrm{Cu}(1)$ & $4 \mathrm{a}$ & $0.8639(1)$ & $0.8639(1)$ & $0.8639(1)$ \\
$\mathrm{Cu}(2)$ & $12 \mathrm{~b}$ & $0.6288(1)$ & $0.6164(1)$ & $0.8781(1)$ \\
$\mathrm{Se}(1)$ & $4 \mathrm{a}$ & $0.2910(1)$ & $0.2910(1)$ & $0.2910(1)$ \\
$\mathrm{Se}(2)$ & $4 \mathrm{a}$ & $0.5387(1)$ & $0.5387(1)$ & $0.5387(1)$ \\
$\mathrm{O}(1)$ & $4 \mathrm{a}$ & $0.7396(3)$ & $0.7396(3)$ & $0.7396(3)$ \\
$\mathrm{O}(2)$ & $12 \mathrm{~b}$ & $0.7193(4)$ & $0.7676(3)$ & $0.2020(3)$ \\
$\mathrm{O}(3)$ & $4 \mathrm{a}$ & $0.9874(3)$ & $0.9874(3)$ & $0.9874(3)$ \\
$\mathrm{O}(4)$ & $12 \mathrm{~b}$ & $0.9369(3)$ & $0.5210(3)$ & $0.2181(3)$ \\
\hline \hline
\end{tabular}

left-handed structure while $x \approx 1-0.137=0.863$ indicates the right-handed enantiomer [6]. Due to the similarity between $\mathrm{Cu}(1)$ in $\mathrm{Cu}_{2} \mathrm{OSeO}_{3}(x=0.8639)$ and $\mathrm{Mn}$ in $\operatorname{MnSi}(x=0.863$ for the right-handed form) we define the observed absolute structure in $\mathrm{Cu}_{2} \mathrm{OSeO}_{3}$ to be right-handed. This definition agrees with that proposed in Ref. [32] which was made on the basis of closeness of the $P 2_{1} 3$ symmetry describing the absolute structure to $P 4_{1} 32$. Such a closeness was first noted in Ref. [2] and also recently mentioned in Ref. [33] for the B20 compounds.

The crystal structure of $\mathrm{Cu}_{2} \mathrm{OSeO}_{3}$ is normally characterized in terms of copper-centered polyhedrons [13]. Here we apply the concept of anion-centered building blocks [34] which provides a rather simple description of the determined crystal structure. In this case, the minimal structural unit is composed of two corner-sharing oxygen-centered tetrahedrons [Fig. 4(a)] that form $\left[\mathrm{O}_{2} \mathrm{Cu}_{7}\right]^{10+}$ dimers. Such a building block orders ferrimagnetically $[13,35]$ with opposite direction of magnetic moments localized on $\mathrm{Cu}(1)$ and $\mathrm{Cu}(2)$ [Fig. 4(b)]. As shown (a)

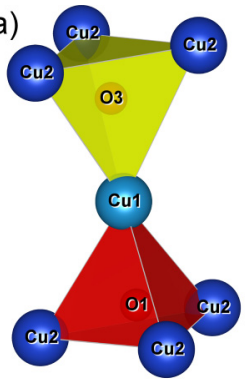

(b)

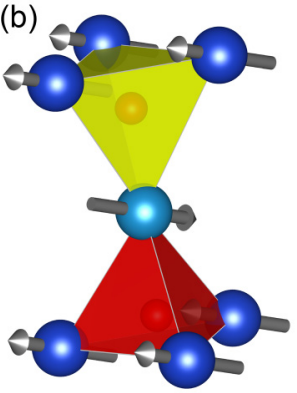

(c)

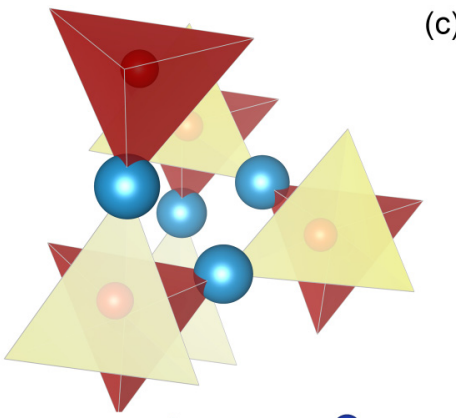

(d)

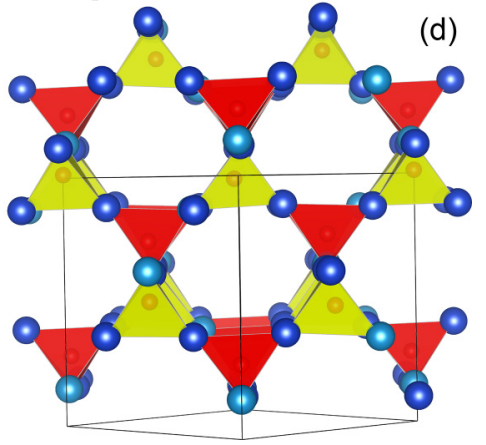

FIG. 4. (Color online) View of a $\left[\mathrm{O}_{2} \mathrm{Cu}_{7}\right]^{10+}$ dimer (a) with the magnetic moments (b) adopted from Refs. [13,35]. These dimers form a spiral along the axis (111) (c). The 3D framework made by the dimers along (110) (d). 
TABLE III. Correlation between the structural $\gamma_{s}$ and magnetic $\gamma_{m}$ chirality in the different compounds with space group $P 2_{1} 3 .+$ and - indicate right- and left-handedness, respectively. $+/-$ or $-/+$ denote cases where both left- and right-handed forms have been observed.

\begin{tabular}{lcccccc}
\hline \hline & $\mathrm{MnSi}$ & $\mathrm{MnGe}$ & $\mathrm{MnFeSi}$ & $\mathrm{FeCoSi}$ & $\mathrm{FeGe}$ & $\mathrm{Cu}_{2} \mathrm{OSeO}_{3}$ \\
\hline$\gamma_{s}$ & $+/-$ & - & $+/-$ & $+/-$ & - & + \\
$\gamma_{m}$ & $+/-$ & - & $+/-$ & $-/+$ & + & + \\
$\gamma_{s} \gamma_{m}$ & + & + & + & - & - & + \\
\hline \hline
\end{tabular}

in Fig. 4(c), the dimers form a screw propagating along the [111] direction of the cubic cell. Additionally, the dimers are connected in a 3D framework [Fig. 4(d)] with vast pores filled by cation-centered tetrahedrons $\mathrm{SeO}_{3}$-lp, where lp stands for lone electron pair [13]. The 3D framework of $\left[\mathrm{O}_{2} \mathrm{Cu}_{7}\right]^{10+}$ dimers carry magnetic properties that can be influenced by additional superexchange paths involving oxygen anions in the $\mathrm{SeO}_{3}$-lp units. These exchange contributions have been discussed in Ref. [35]; such an intrinsic coupling between magnetic framework and polar entities filling the pores could even serve as a pathway for a magnetoelectric response.

Similarly to the B20 compounds, one could expect an inherent link between the structural chirality of the 3D dimer framework and the chirality of the magnetic structure. All measurements concerning the magnetic and structural chiralities done so far on $P 2{ }_{1} 3$ compounds [3-9] are summarized in Table III. Despite very different transport and magnetotransport properties, these compounds display similar magnetic phase diagrams, have the same space group, and have magnetic and structural chiralities correlated with each other.

Notably, the structure of $\mathrm{Cu}_{2} \mathrm{OSeO}_{3}$ with the same set of coordinates as present in Table II has also been denoted as right-handed in the Ref. [32]. There, a multishell Heisenberg-like model was implemented for calculating the expected sign of the DMI based on the chirality of the crystal structure [11]. The expectations of the model successfully describe the relationship between the two chiralities for $\mathrm{MnSi}$ observed experimentally (Table III) [11,32] yet predicts the opposite chiral interrelation for the case of $\mathrm{Cu}_{2} \mathrm{OSeO}_{3}$; the right-handed structure is expected to conjugate with the left-handed magnetic helix [32]. The experimental findings reported here show that this conjecture does not meet reality and so calls for a theoretical revision. A possible reconciliation between theory and experiment may be achieved by including more coordinating shells in the theoretical analysis.
In this Rapid Communication we have shown the combination of polarized SANS and single crystal diffraction of synchrotron radiation to provide unambiguous information concerning the chiral senses of magnetic and crystal structures. For $\mathrm{Cu}_{2} \mathrm{OSeO}_{3}$ this powerful combination of experimental techniques has been applied for the first time. Similarly to monosilicide and monogermanide $3 d$-metal families, $\mathrm{Cu}_{2} \mathrm{OSeO}_{3}$ is also found to be enantiopure for both magnetic and crystal structures. These observations indicate rather a high energy barrier to separate the two enantiopure domains, and that a low density of domain walls and larger domains are energetically more favorable.

We have found that the crystal and magnetic structures for $\mathrm{Cu}_{2} \mathrm{OSeO}_{3}$ have the same chirality. The same relationship between two chiralities was observed in $\mathrm{MnSi}, \mathrm{Mn}_{1-x} \mathrm{Fe}_{x} \mathrm{Si}$, and $\mathrm{MnGe}$. At variance, $\mathrm{FeGe}$ and $\mathrm{Fe}_{1-x} \mathrm{Co}_{x} \mathrm{Si}$ always show the opposite magnetic and structural chiralities. We expect that combining these findings will constrain new calculations concerned with a general microscopic understanding of the coupling between the chiralities. The observed relationship between structural and magnetic chiralities both for metals (MnSi [3-5,9], FeGe [8], MnGe [8] and solid solutions $\mathrm{Fe}_{1-x} \mathrm{Co}_{x} \mathrm{Si}[5,7,9], \mathrm{Mn}_{1-x} \mathrm{Fe}_{x} \mathrm{Si}$ [6], $\mathrm{Mn}_{1-x} \mathrm{Fe}_{x} \mathrm{Ge}$ [8]) and an insulator $\left(\mathrm{Cu}_{2} \mathrm{OSeO}_{3}\right)$ allows us to conclude that conduction electrons play no role in a possible common mechanism describing the magnetolattice interaction.

Stringent tests of theories describing the magnetolattice interaction will also need to describe the chiral couplings in materials with space groups different from $P 2_{1} 3$. Prominent examples include $\mathrm{Cr}_{1 / 3} \mathrm{NbS}_{2}$ (space group $P 6_{3} 22$ ) [36] and the trigonal chiral compound $\mathrm{Ba}_{3} \mathrm{NbFe}_{3} \mathrm{Si}_{2} \mathrm{O}_{14}$ (space group P321) [37-39], which displays structurally enantiopure crystals with single magnetic chirality. The accumulation of experimental data on the coupling between structural and magnetic chiralities is a necessary condition not only for understanding the underlying microscopic mechanisms but also for developing the control of exotic magnetic textures as is widely discussed in the context of potential spintronics applications $[18,40]$. This mostly missing experimental information on the chiral coupling between structure and magnetism in magnetoelectrics is especially important for the so-called skyrmion phase which could potentially offer new energy-efficient spintronics options [17].

We acknowledge Dr. Alexey Bosak for the sample preparation and Prof. Vladimir Dmitriev for numerous stimulating discussions.
[1] J. Jensen and A. R. Mackintosh, Rare Earth Magnetism: Structures and Excitations (Clarendon Press, Oxford, 1991).

[2] V. Dmitriev, D. Chernyshov, S. Grigoriev, and V. Dyadkin, J. Phys.: Condens. Matter 24, 366005 (2012).

[3] M. Tanaka, H. Takayoshi, M. Ishida, and Y. Endoh, J. Phys. Soc. Jpn. 54, 2970 (1985).

[4] M. Ishida, Y. Endoh, S. Mitsuda, Y. Ishikawa, and M. Tanaka, J. Phys. Soc. Jpn. 54, 2975 (1985).
[5] S. V. Grigoriev, D. Chernyshov, V. A. Dyadkin, V. Dmitriev, S. V. Maleyev, E. V. Moskvin, D. Menzel, J. Schoenes, and H. Eckerlebe, Phys. Rev. Lett. 102, 037204 (2009).

[6] S. V. Grigoriev, D. Chernyshov, V. A. Dyadkin, V. Dmitriev, E. V. Moskvin, D. Lamago, T. Wolf, D. Menzel, J. Schoenes, S. V. Maleyev, and H. Eckerlebe, Phys. Rev. B 81, 012408 (2010). 
[7] V. A. Dyadkin, S. V. Grigoriev, D. Menzel, D. Chernyshov, V. Dmitriev, J. Schoenes, S. V. Maleyev, E. V. Moskvin, and H. Eckerlebe, Phys. Rev. B 84, 014435 (2011).

[8] S. V. Grigoriev, N. M. Potapova, S.-A. Siegfried, V. A. Dyadkin, E. V. Moskvin, V. Dmitriev, D. Menzel, C. D. Dewhurst, D. Chernyshov, R. A. Sadykov, L. N. Fomicheva, and A. V. Tsvyashchenko, Phys. Rev. Lett. 110, 207201 (2013).

[9] D. Morikawa, K. Shibata, N. Kanazawa, X. Z. Yu, and Y. Tokura, Phys. Rev. B 88, 024408 (2013).

[10] P. Båk and M. H. Jensen, J. Phys. C 13, L881 (1980).

[11] V. A. Chizhikov and V. E. Dmitrienko, Phys. Rev. B 88, 214402 (2013).

[12] K. Kohn, J. Phys. Soc. Jpn. 42, 2065 (1977).

[13] Jan-Willem G. Bos, C. V. Colin, and T. T. M. Palstra, Phys. Rev. B 78, 094416 (2008).

[14] S. Seki, S. Ishiwata, and Y. Tokura, Phys. Rev. B 86, 060403 (2012).

[15] T. Adams, A. Chacon, M. Wagner, A. Bauer, G. Brandl, B. Pedersen, H. Berger, P. Lemmens, and C. Pfleiderer, Phys. Rev. Lett. 108, 237204 (2012).

[16] S. Seki, J.-H. Kim, D. S. Inosov, R. Georgii, B. Keimer, S. Ishiwata, and Y. Tokura, Phys. Rev. B 85, 220406 (2012).

[17] S. Seki, X. Z. Yu, S. Ishiwata, and Y. Tokura, Science 336, 198 (2012).

[18] J. S. White, I. Levatić, A. A. Omrani, N. Egetenmeyer, K. Prša, I. Živković, J. L. Gavilano, J. Kohlbrecher, M. Bartkowiak, H. Berger, and H. M. Rønnow, J. Phys.: Condens. Matter 24, 432201 (2012).

[19] G. Meunier and M. Bertaud, J. Appl. Crystallogr. 9, 364 (1976).

[20] H. Effenberger and F. Pertlik, Monatsh. Chem. 117, 887 (1985).

[21] H. D. Flack, Acta Crystallogr. Sec. A 39, 876 (1983).

[22] H. D. Flack and G. Bernardinelli, Acta Crystallogr. Sec. A 55, 908 (1999).

[23] U. Shmueli, M. Schiltz, and H. D. Flack, Acta Crystallogr. Sec. A 64, 476 (2008).

[24] S. V. Maleyev, Phys. Rev. Lett. 75, 4682 (1995).
[25] V. P. Plakhty, W. Schweika, T. Brückel, J. Kulda, S. V. Gavrilov, L.-P. Regnault, and D. Visser, Phys. Rev. B 64, 100402 (2001).

[26] S. V. Grigoriev, Y. O. Chetverikov, D. Lott, and A. Schreyer, Phys. Rev. Lett. 100, 197203 (2008).

[27] S. V. Grigoriev, V. A. Dyadkin, D. Menzel, J. Schoenes, Y. O. Chetverikov, A. I. Okorokov, H. Eckerlebe, and S. V. Maleyev, Phys. Rev. B 76, 224424 (2007).

[28] G. Shirane, R. Cowley, C. Majkrzak, J. B. Sokoloff, B. Pagonis, C. H. Perry, and Y. Ishikawa, Phys. Rev. B 28, 6251 (1983).

[29] S. V. Grigoriev, S. V. Maleyev, A. I. Okorokov, Yu. O. Chetverikov, P. Böni, R. Georgii, D. Lamago, H. Eckerlebe, and K. Pranzas, Phys. Rev. B 74, 214414 (2006).

[30] See Supplemental Material at http://link.aps.org/supplemental/ 10.1103/PhysRevB.89.140409 for the crystallographic information file (CIF).

[31] H. D. Flack and G. Bernardinelli, J. Appl. Crystallogr. 33, 1143 (2000).

[32] V. A. Chizhikov and V. E. Dmitrienko, arXiv:1305.5382v1.

[33] J. Kübler, B. Yan, and C. Felser, Europhys. Lett. 104, 30001 (2013).

[34] S. V. Krivovichev, O. Mentré, O. I. Siidra, M. Colmont, and S. K. Filatov, Chem. Rev. 113, 6459 (2013).

[35] I. Živković, D. Pajić, T. Ivek, and H. Berger, Phys. Rev. B 85, 224402 (2012).

[36] Y. Togawa, T. Koyama, K. Takayanagi, S. Mori, Y. Kousaka, J. Akimitsu, S. Nishihara, K. Inoue, A. S. Ovchinnikov, and J. Kishine, Phys. Rev. Lett. 108, 107202 (2012).

[37] K. Marty, V. Simonet, E. Ressouche, R. Ballou, P. Lejay, and P. Bordet, Phys. Rev. Lett. 101, 247201 (2008).

[38] V. Scagnoli, S. W. Huang, M. Garganourakis, R. A. de Souza, U. Staub, V. Simonet, P. Lejay, and R. Ballou, Phys. Rev. B 88, 104417 (2013).

[39] V. Simonet, M. Loire, and R. Ballou, Eur. Phys. J. Spec. Top. 213, 5 (2012).

[40] C. Pfleiderer, A. Neubauer, S. Mühlbauer, F. Jonietz, M. Janoschek, S. Legl, R. Ritz, W. Münzer, C. Franz, P. G. Niklowitz, T. Keller, R. Georgii, P. Böni, B. Binz, A. Rosch, U. K. Rossler, and A. N. Bogdanov, J. Phys.: Condens. Matter 21, 164215 (2009). 